MATHEMATICS OF COMPUTATION

Volume 76, Number 259, July 2007, Pages 1493-1498

S 0025-5718(07)01962-X

Article electronically published on January 24, 2007

\title{
K3 SURFACES WITH PICARD NUMBER THREE AND CANONICAL VECTOR HEIGHTS
}

\author{
ARTHUR BARAGAR AND RONALD VAN LUIJK
}

\begin{abstract}
In this paper we construct the first known explicit family of K3 surfaces defined over the rationals that are proved to have geometric Picard number 3. This family is dense in one of the components of the moduli space of all polarized K3 surfaces with Picard number at least 3. We also use an example from this family to fill a gap in an earlier paper by the first author. In that paper, an argument for the nonexistence of canonical vector heights on K3 surfaces of Picard number 3 was given, based on an explicit surface that was not proved to have Picard number 3 . We redo the computations for one of our surfaces and come to the same conclusion.
\end{abstract}

\section{INTRODUCTION}

The main result of this paper is the construction of an infinite family of K3 surfaces with geometric Picard number 3 that is dense in a component of the moduli space of polarized K3 surfaces of Picard number at least 3. This family is given by smooth $(2,2,2)$-forms in $\mathbb{P}^{1} \times \mathbb{P}^{1} \times \mathbb{P}^{1}$. The methods we use are similar to those in [5].

The second intent of this paper is to fill a gap in an argument in [1], which was pointed out by Yuri Tschinkel in the review of the paper and privately by Bert van Geemen. In that paper, the first author gave convincing numerical evidence for the nonexistence of canonical vector heights on K3 surfaces of Picard number 3. Though the Picard number of the surface used in 1 is at least 3 , it was not proved to equal 3. Instead of proving equality, we redo the calculations using one of the surfaces found here. Again, we come to the conclusion that canonical vector heights do not exist.

We thank MSRI for their hospitality and support and UNLV for their support of the first author during his sabbatical semester.

\section{K3 SURFACES With PiCARD NUMBer THREE}

Let $k$ be a field with a fixed algebraic closure $\bar{k}$. Let $X$ be a smooth surface over $k$ in $\mathbb{P}^{1} \times \mathbb{P}^{1} \times \mathbb{P}^{1}$, given by a $(2,2,2)$-form. Then $X$ is a K3 surface, which implies that linear, algebraic, and numerical equivalence all coincide. This means that the Picard group Pic $\bar{X}$ and the Néron-Severi group NS $\bar{X}$ of $\bar{X}=X_{\bar{k}}$ are naturally isomorphic, finitely generated, and free. Their rank is called the (geometric) Picard number of

Received by the editor February 22, 2006 and, in revised form, July 14, 2006.

2000 Mathematics Subject Classification. Primary 14G40, 11G50, 14J28, 14C22.

Key words and phrases. K3 surfaces, canonical vector heights, heights, Picard numbers.

The first author is supported in part by NSF grant DMS-0403686.

(C)2007 American Mathematical Society

Reverts to public domain 28 years from publication 
$X$. By the Hodge Index Theorem, the intersection pairing gives this group the structure of a lattice with signature $(1, \operatorname{rk} N S \bar{X}-1)$. For detailed definitions of all these notions, see [5]. Note however, that in this paper the Picard number always refers to the geometric Picard number.

For $i=1,2,3$, let $\pi_{i}: X \rightarrow \mathbb{P}^{1}$ be the projection from $X$ to the $i$-th copy of $\mathbb{P}^{1}$ in $\mathbb{P}^{1} \times \mathbb{P}^{1} \times \mathbb{P}^{1}$. Let $D_{i}$ denote the divisor class represented by a fiber of $\pi_{i}$. We find $D_{i} \cdot D_{j}=2$ for $i \neq j$ and since any two different fibers of $\pi_{i}$ are disjoint, we have $D_{i}^{2}=0$. It follows that the intersection matrix $\left[D_{i} \cdot D_{j}\right]_{i, j}$ has rank 3 , so the $D_{i}$ generate a subgroup of the Néron-Severi group $\mathrm{NS}(\bar{X})$ of rank 3. Our goal is to find explicit examples for which the rank of $\mathrm{NS}(\bar{X})$ equals 3.

Let $x, y$, and $z$ denote the affine coordinates of $\mathbb{A}^{1}$ inside the three copies of $\mathbb{P}^{1}$ in $\mathbb{P}^{1} \times \mathbb{P}^{1} \times \mathbb{P}^{1}$. Set

$$
\begin{aligned}
& G_{1}=-y^{2} z^{2}+3 y^{2} z+2 y^{2}-2 y z^{2}+3 y z+3 y+2 z^{2}+2 z-1, \\
& G_{2}=2 y^{2} z^{2}+3 y^{2} z+3 y^{2}+2 y z^{2}+2 y z+3 z^{2}+z+2, \\
& G_{3}=y^{2} z+y^{2}+y+z^{2}+z, \\
& L_{1}=y z-y-z \\
& L_{2}=y z+1 .
\end{aligned}
$$

Theorem 2.1. For any $H \in \mathbb{Z}[x, y, z]$ with degree at most 2 in each of $x, y$, and $z$, the surface $Y \subset \mathbb{P}^{1} \times \mathbb{P}^{1} \times \mathbb{P}^{1}$ with affine equation

$$
G_{1} x^{2}+G_{2} x+3 G_{3}-2 L_{1} L_{2}=6 H
$$

is a smooth $\mathrm{K} 3$ surface. The Picard number of $Y_{\overline{\mathbb{Q}}}$ equals 3 .

Remark 2.2. A surface in $\mathbb{P}^{1} \times \mathbb{P}^{1} \times \mathbb{P}^{1}$ given by a $(2,2,2)$-form $F$ is determined by the coefficients of 27 monomials. Since the equation $F=0$ is homogeneous, this gives a 26-dimensional family of K3 surfaces. After dividing out by the 3-dimensional automorphism groups of the three copies of $\mathbb{P}^{1}$, this leaves a 17 -dimensional family of isomorphism classes. Note that the dimension of the moduli space of polarized K3 surfaces whose Néron-Severi group contains a prescribed lattice of rank $\rho$ equals $20-\rho$. For the lattice $\Lambda$ of rank $\rho=3$ generated by the $D_{i}$ as described above, this reflects the fact that the family of smooth surfaces in $\mathbb{P}^{1} \times \mathbb{P}^{1} \times \mathbb{P}^{1}$ given by $(2,2,2)$ forms contains an open subset that is birationally equivalent with the moduli space of K3 surfaces $X$ together with an embedding of the lattice $\Lambda$ into NS $X$. The freedom of choice for $H$ shows that the surfaces of Theorem 2.1 form a set that is dense in this moduli space in both the Zariski and the real analytic topology.

To bound the Picard number, we use the method described in [5. We first state some results and notation that we will use. Let $X$ be any smooth surface over a number field $K$ and let $\mathfrak{p}$ be a prime of good reduction with residue field $k$. Let $\mathfrak{X}$ be an integral model for $X$ over the localization $\mathcal{O}_{\mathfrak{p}}$ of the ring of integers $\mathcal{O}$ of $K$ at $\mathfrak{p}$ for which the reduction is smooth. Let $k^{\prime}$ be any extension field of $k$. Then by abuse of notation we will write $X_{k^{\prime}}$ for $\mathfrak{X} \times_{\operatorname{Spec} \mathcal{O}_{\mathfrak{p}}} \operatorname{Spec} k^{\prime}$.

Proposition 2.3. Let $X$ be a smooth surface over a number field $K$ and let $\mathfrak{p}$ be a prime of good reduction with residue field $k$. Let $l$ be a prime not dividing $q=\# k$. Let $F$ denote the automorphism on $H_{\text {ét }}^{2}\left(X_{\bar{k}}, \mathbb{Q}_{l}\right)(1)$ induced by q-th power Frobenius. 
Then there are natural injections

$$
\mathrm{NS}\left(X_{\bar{K}}\right) \otimes \mathbb{Q}_{l} \hookrightarrow \mathrm{NS}\left(X_{\bar{k}}\right) \otimes \mathbb{Q}_{l} \hookrightarrow H_{\text {ét }}^{2}\left(X_{\bar{k}}, \mathbb{Q}_{l}\right)(1),
$$

that respect the intersection pairing and the action of Frobenius, respectively. The rank of $\operatorname{NS}\left(X_{\bar{k}}\right)$ is at most the number of eigenvalues of $F$ that are roots of unity, counted with multiplicity.

Proof. See [4, Prop. 6.2 and Cor. 6.4. Note that in the referred corollary, Frobenius acts on the cohomology group $H_{\text {èt }}^{2}\left(X_{\bar{k}}, \mathbb{Q}_{l}\right)$ without a twist. Therefore, the eigenvalues are scaled by a factor $q$.

Proof of Theorem 2.1. Fix a polynomial $H$ and the corresponding surface $Y$ as in Theorem 2.1, We write $Y_{p}$ and $\bar{Y}_{p}$ for $Y_{\mathbb{F}_{p}}$ and $Y_{\overline{\mathbb{F}}_{p}}$, respectively. Note that $Y_{p}$ does not depend on $H$ for $p=2$ and $p=3$. One easily checks that $Y_{p}$ is smooth for $p=2,3$, so $Y$ itself is smooth and $Y$ has good reduction at 2 and 3. Since $Y$ is a smooth surface in $\mathbb{P}^{1} \times \mathbb{P}^{1} \times \mathbb{P}^{1}$ given by a $(2,2,2)$-form, it is a K3 surface. Both $Y_{2}$ and $Y_{3}$ contain a fourth divisor class that is linearly independent of the earlier described classes $D_{i}$ for $i=1,2,3$. On $Y_{2}$ we have the curve $C_{2}$ parameterized by ([x:1], [1:0], [1:1]). On $Y_{3}$ we have the curve $C_{3}$ given by $x=L_{1}=0$. For $p=2,3$, let $\Lambda_{p}$ denote the sublattice of the Néron-Severi group of $\bar{Y}_{p}$ generated by $D_{1}, D_{2}, D_{3}$, and $C_{p}$. The intersection matrices associated to the sequences of classes $\left\{D_{1}, D_{2}, D_{3}, C_{2}\right\}$ and $\left\{D_{1}, D_{2}, D_{3}, C_{3}\right\}$ are

$$
\left[\begin{array}{cccc}
0 & 2 & 2 & 1 \\
2 & 0 & 2 & 0 \\
2 & 2 & 0 & 0 \\
1 & 0 & 0 & -2
\end{array}\right] \quad \text { and } \quad\left[\begin{array}{cccc}
0 & 2 & 2 & 0 \\
2 & 0 & 2 & 1 \\
2 & 2 & 0 & 1 \\
0 & 1 & 1 & -2
\end{array}\right] \text {, }
$$

so $\Lambda_{2}$ and $\Lambda_{3}$ have discriminants -28 and -32 , respectively. We will now show that the Picard numbers of $\bar{Y}_{2}$ and $\bar{Y}_{3}$ both equal 4. Almost all fibers of the fibration $\pi_{1}$ are smooth curves of genus 1. Using MAGMA, we counted the number of points over small fields fiber by fiber. The total numbers of points are given in Table 1 ,

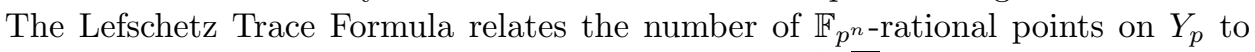
the traces of the $p^{n}$-th power Frobenius acting on $H_{\text {ét }}^{i}\left(\bar{Y}_{p}, \mathbb{Q}_{l}\right)(1)$ for $i=0, \ldots, 4$

TABLE 1. Number of points over some finite fields.

\begin{tabular}{|l|l|l|}
\hline$n$ & $\# Y_{2}\left(\mathbb{F}_{2^{n}}\right)$ & $\# Y_{3}\left(\mathbb{F}_{3^{n}}\right)$ \\
\hline 1 & 13 & 17 \\
2 & 25 & 107 \\
3 & 85 & 848 \\
4 & 289 & 6719 \\
5 & 1153 & 60632 \\
6 & 4273 & 536564 \\
7 & 16897 & 4793855 \\
8 & 65025 & 43091783 \\
9 & 266305 & 387501194 \\
10 & 1050625 & \\
\hline
\end{tabular}


by

$$
\# Y_{p}\left(\mathbb{F}_{p^{n}}\right)=\sum_{i=0}^{4}\left(-p^{n / 2}\right)^{i} \cdot\left(\text { trace of } p^{n} \text {-th power Frobenius on } H_{\text {ét }}^{i}\left(\bar{Y}_{p}, \mathbb{Q}_{l}\right)(1)\right) .
$$

Normally this is phrased in terms of the cohomology groups without the twist. For K3 surfaces we have $\operatorname{dim} H^{i}=1,0,22,0,1$ for $i=0,1,2,3,4$, respectively. Since the action for $i \neq 2$ is trivial, from the numbers in Table 1 we can compute the traces of powers of the automorphism $F_{p}$ on $H_{\text {ét }}^{2}\left(\bar{Y}_{p}, \mathbb{Q}_{l}\right)(1)$ that is induced by $p$-th power Frobenius. We find $p^{n} \cdot \operatorname{Tr} F_{p}^{n}=\# Y_{p}\left(\mathbb{F}_{p^{n}}\right)-p^{2 n}-1$. For $p=2,3$, let $W_{p}$ denote the quotient of $H_{\text {ét }}^{2}\left(\bar{Y}_{p}, \mathbb{Q}_{l}\right)(1)$ by the image $V_{p}$ of $\Lambda_{p} \otimes \mathbb{Q}_{l}$ under the second homomorphism in Proposition 2.3. and let $\Phi_{p}$ denote the action of Frobenius on $W_{p}$. Since $F_{p}$ acts trivially on $V_{p}$, we have $\operatorname{Tr} \Phi_{p}^{n}=\operatorname{Tr} F_{p}^{n}-\operatorname{Tr} F_{p}^{n} \mid V_{p}=\operatorname{Tr} F_{p}^{n}-4$ for all $n \geq 0$, and $f_{F_{p}}=f_{F_{p} \mid V_{p}} \cdot f_{\Phi_{p}}=(t-1)^{4} f_{\Phi_{p}}$, where $f_{T}$ stands for the characteristic polynomial of the linear operator $T$. From the traces of the first $s>0$ powers of a linear operator one can derive the first $s$ coefficients of its characteristic polynomial (see [5], Lemma 2.4). Once enough coefficients of $f_{\Phi_{p}}$ are computed, the full polynomial $f_{\Phi_{p}}$ follows from the functional equation $f_{\Phi_{p}}(1 / x)=$ $\pm x^{-\operatorname{dim} W_{p}} f_{\Phi_{p}}(x)$. Putting all this together, we find $f_{F_{p}}=\frac{1}{p}(t-1)^{4} f_{\Phi_{p}}$ with

$$
\begin{aligned}
f_{\Phi_{2}}= & 2 t^{18}+2 t^{16}+t^{15}+2 t^{14}+t^{13}+2 t^{12}+t^{11}+3 t^{10} \\
& +3 t^{8}+t^{7}+2 t^{6}+t^{5}+2 t^{4}+t^{3}+2 t^{2}+2, \\
f_{\Phi_{3}}= & 3 t^{18}+5 t^{17}+6 t^{16}+5 t^{15}+5 t^{14}+6 t^{13}-6 t^{11}-5 t^{10} \\
& -6 t^{9}-5 t^{8}-6 t^{7}+6 t^{5}+5 t^{4}+5 t^{3}+6 t^{2}+5 t+3 .
\end{aligned}
$$

Note that the coefficient of $t^{9}$ in $f_{\Phi_{2}}$ is zero, so we used the number of points over $\mathbb{F}_{2}^{10}$ to compute the coefficient of $t^{8}$, from which we determined the sign of the functional equation to be positive. Both $f_{\Phi_{p}}$ are irreducible. Their roots are not integral and therefore they are not roots of unity. By Proposition 2.3 we find that the Picard numbers of $\bar{Y}_{2}$ and $\bar{Y}_{3}$ are both bounded by 4 , so they are equal to 4 and $\Lambda_{p}$ has finite index in $\operatorname{NS}\left(\bar{Y}_{p}\right)$ for $p=2,3$. It is well known that if $\Lambda^{\prime}$ is a sublattice of finite index in the lattice $\Lambda$, then we have

$$
\operatorname{disc} \Lambda^{\prime}=\left[\Lambda: \Lambda^{\prime}\right]^{2} \operatorname{disc} \Lambda \text {. }
$$

Thus, up to a square factor, the discriminants of $\mathrm{NS}\left(\bar{Y}_{2}\right)$ and $\mathrm{NS}\left(\bar{Y}_{3}\right)$ are equal to -28 and -32 , respectively. From the first injection of Proposition 2.3 we find $\operatorname{rk} \mathrm{NS}(\bar{Y}) \leq 4$. Suppose we had equality. Then the lattice $\mathrm{NS}(\bar{Y})$ would be isomorphic to a sublattice of finite index in $\mathrm{NS}\left(\bar{Y}_{p}\right)$ for both $p=2$ and $p=3$. By (1), this implies that up to a square factor, the discriminant of $\mathrm{NS}(\bar{Y})$ is equal to both -28 and -32 . This contradicts the fact that -28 and -32 do not differ by a square factor. We therefore conclude that equality does not hold and we have rk NS $(\bar{Y}) \leq 3$. Since the classes $D_{1}, D_{2}$, and $D_{3}$ are linearly independent, we deduce $\operatorname{rk} \mathrm{NS}(\bar{Y})=3$.

\section{Nonexistence of CANONICAL VECTOR Heights}

One way to fill the gap in [1] would be to prove that the surface used there has Picard number 3. The only method currently known to do this is the method used in the previous section. It requires two primes of good reduction for which the reductions have Picard number 4 . Modulo 2 and 3 the Picard numbers turn 
TABLE 2. Estimates for $\hat{h}_{E_{i j k}}\left(P_{0}\right)$ for the permutations $(i, j, k)$ of $(1,2,3)$.

\begin{tabular}{|c|c|c|c|c|c|c|}
\hline$n$ & $(1,2,3)$ & $(1,3,2)$ & $(2,1,3)$ & $(2,3,1)$ & $(3,1,2)$ & $(3,2,1)$ \\
\hline 1 & 0.3438678 & 1.0306631 & 1.7914641 & 2.0624775 & 1.7723601 & 1.6340533 \\
2 & 0.4711022 & 1.0326396 & 1.8311032 & 2.1288087 & 1.8613679 & 1.7950761 \\
3 & 0.4745990 & 1.0365615 & 1.8328300 & 2.1330968 & 1.8675712 & 1.7982461 \\
4 & 0.4747015 & 1.0364020 & 1.8329385 & 2.1332594 & 1.8679417 & 1.7986626 \\
5 & 0.4746928 & 1.0364196 & 1.8329585 & 2.1332721 & 1.8679467 & 1.7986781 \\
\hline
\end{tabular}

out to be 16 and 6, respectively (depending on Tate's conjecture). The computations required to calculate the Picard number modulo larger primes are currently beyond our ability as counting points takes too much time. We therefore redo the calculations in [1] for one of the surfaces of Theorem 2.1.

For the remainder of this section, let $Y$ denote the surface associated to $H=0$ as in Theorem 2.1. Then $Y$ has Picard number 3. As in [1, we let $\sigma_{i}$ denote the involution associated to the 2 -to-1 projection $Y \rightarrow \mathbb{P}^{1} \times \mathbb{P}^{1}$ along the $i$-th copy of $\mathbb{P}^{1}$ in $\mathbb{P}^{1} \times \mathbb{P}^{1} \times \mathbb{P}^{1}$, and for $i, j, k \in\{1,2,3\}$, we set $\sigma_{i j k}=\sigma_{i} \sigma_{j} \sigma_{k}$. Let $\mathcal{D}^{*}=\left\{D_{1}^{*}, D_{2}^{*}, D_{3}^{*}\right\}$ be the basis that is dual to the basis $\mathcal{D}=\left\{D_{1}, D_{2}, D_{3}\right\}$ of $\mathrm{NS}(\bar{Y}) \otimes \mathbb{R}$. Let the heights $h_{D_{i}}$ be defined by $\pi_{i}$ and the usual logarithmic height on $\mathbb{P}^{1}(\mathbb{Q})$. Then $h_{D_{i}}$ is a Weil height associated to $D_{i}$ and

$$
\mathbf{h}=\sum_{i=1}^{3} h_{D_{i}} D_{i}^{*}
$$

is a vector height, so for every divisor class $E \in \mathrm{NS}(\bar{Y}) \otimes \mathbb{R}$, a Weil height $h_{E}$ associated to $E$ is up to $O(1)$ given by $P \mapsto \mathbf{h}(P) \cdot E$.

Vector heights also satisfy the property that $\mathbf{h}(\sigma P)=\sigma_{*} \mathbf{h}(P)+\mathbf{O}(1)$ for all $\sigma \in \operatorname{Aut}(Y)$ and all $P \in Y(\mathbb{Q})$, where $\sigma_{*}$ acts on $\operatorname{NS}(\bar{Y}) \otimes \mathbb{R}$ and bounds on the error term may depend on $\sigma$ but not on $P$. We say a vector height $\hat{\mathbf{h}}$ is a canonical vector height if the error term is zero for all $\sigma$ and all $P$.

Suppose $\sigma$ is an automorphism of $Y$ and that the pullback $\sigma^{*}$ acting on $\operatorname{NS}(\bar{Y}) \otimes \mathbb{R}$ has a real eigenvalue $\omega>1$ with associated eigenvector $E$. Silverman 2] defined the canonical height (with respect to $\sigma$ ) to be

$$
\hat{h}_{E}(P)=\lim _{n \rightarrow \infty} \omega^{-n} h_{E}\left(\sigma^{n} P\right) .
$$

The canonical height $\hat{h}_{E}$ and the canonical vector height $\hat{\mathbf{h}}$ satisfy the relation $\hat{h}_{E}=\hat{\mathbf{h}} \cdot E($ see 1$\left.]\right)$.

Set $\gamma=\frac{1}{2}(1+\sqrt{5})$. Then $\alpha$ and $\omega$ in [1] are equal to $\gamma^{2}$ and $\gamma^{6}$, respectively. Suppose $(i, j, k)$ is a permutation of $(1,2,3)$. The eigenvector $E_{i j k}$ of $\sigma_{i}^{*} \sigma_{j}^{*} \sigma_{k}^{*}=\sigma_{k j i}^{*}$ associated to the eigenvalue $\omega$, as defined in [1], equals $\frac{1}{2} \gamma\left(-D_{i}+\gamma D_{j}+\gamma^{2} D_{k}\right)$. Set $P_{0}=([0: 1],[0: 1],[0: 1])$. Table 2 contains the estimates $\omega^{-n} \mathbf{h}\left(\sigma_{k j i}^{n} P_{0}\right) \cdot E_{i j k}$ of the canonical height $\hat{h}_{E_{i j k}}\left(P_{0}\right)$ (canonical with respect to $\sigma_{k j i}$ ) for all permutations $(i, j, k)$ and $n \in\{1, \ldots, 5\}$.

These estimates appear to converge geometrically, as expected. We believe, without rigorous proof, that the estimates of the canonical heights for $n=5$ are correct up to an error of at most 0.0001 and are probably correct up to 0.00001 . The six estimates in Table 2 give us six linear equations in the three components of 
$\hat{\mathbf{h}}(P)$, leading to a contradiction. More precisely, following an argument similar to that presented in [1, we conclude the following, which gives evidence against the existence of a canonical vector height on $Y$.

Theorem 3.1. If the estimates $\omega^{-5} h_{E_{i j k}}\left(\sigma_{k j i}^{5} P_{0}\right)$ in Table 2 are equal to the canonical heights $\hat{h}_{E_{i j k}}\left(P_{0}\right)$ up to an absolute error of at most 0.1 , then the surface $Y$ does not admit a canonical vector height.

\section{REFERENCES}

[1] A. Baragar, Canonical vector heights on $\mathrm{K} 3$ surfaces with Picard number three - an argument for non-existence, Math. Comput. (248) 73 (2004), 2019-2025. MR2005e:14058

[2] J. Silverman, Rational points on K3 surfaces: A new canonical height, Invent. Math. 105 (1991), 347 - 373. MR92k:14025

[3] J. Tate, Algebraic cycles and poles of zeta functions, Arithmetical Algebraic Geometry, O.F.G. Schilling, ed. (1965), 93-110. MR37:1371

[4] R. van Luijk, An elliptic K3 surface associated to Heron triangles, J. Number Theory (to appear); available at arXiv:math.AG/0411606 (2004).

[5] R. van Luijk, K3 surfaces with Picard number one and infinitely many rational points, preprint, available at arXiv:math.AG/0506416 (2005).

Department of Mathematical Sciences, University of Nevada las Vegas, Las Vegas, NEVADA 89154-4020

E-mail address: baragar@unlv.nevada.edu

Mathematical Sciences Research Institute, 17 Gauss Way, Berkeley, California 94720-5070

Current address: Department of Mathematics, Simon Fraser University, 8888 University Drive, Burnaby, BC V5A 1S6, Canada

E-mail address: rmluijk@gmail.com 